\title{
Valores semántico-pragmáticos de aún
}

\author{
Laura D. Ferrari - Universidad de Buenos Aires/ \\ Universidad Nacional de General Sarmiento \\ Iferrari1@live.com.ar \\ Mabel Giammatteo - Universidad de Buenos Aires/Universidad del Salvador \\ ggiammat@gmail.com
}

Rebut / Received: 1-9-15

Acceptat / Accepted: 22-1-16

Resum. Valors semanticopragmàtics d'aún. Els diccionaris i les gramàtiques solen distingir entre aún temporal i aun amb valors de tipus modal-concessiu. Segons aquesta caracterització, el primer és commutable per todavía i porta accent gràfic mentre que el segon no s’accentua i és reemplaçable per hasta, incluso o siquiera. La multiplicitat de funcions i valors semàntics d'aún, ja advertida per Bello (\$1217), ha portat alguns autors a incloure’l en el grup de les “partícules” (Pavon Lucero 1999), una metaclasse que engloba preposicions, adverbis i conjuncions. Altres estudis (Sánchez López 1999, Leonetti 2007 i NGRALE 2009) l'han ubicat dins la subclasse dels focalitzadors, la funció dels quals és posar de relleu l'estructura informativa de l'oració. En aquest treball, ens interessa plantejar que totes dues variants -aún temporal / aun concessiuno representen un contrast dicotòmic sinó que comparteixen un valor quantificacional que es vincula amb el caràcter escalar que també té aquest mot. Aquesta similitud s'evidencia en els casos en què aquesta partícula incideix sobre una comparació i resulta possible tant la substitució per todavía com per incluso.

Paraules clau: partícules, focalitzadors, estructura informativa, quantificació.

Abstract. Semantic and pragmatic values of aún. Spanish dictionaries and grammar textbooks usually describe the word aún (even) as an expression of time and aun as an expression of modality (concession). In the first case, it is interchangeable with todavia (yet) and it carries an

1. Una versión preliminar de este trabajo (Ferrari y Giammatteo, en prensa), fue leída en las II Jornadas Internacionales Beatriz Lavandera. Sociolingüística y Análisis del Discurso, realizadas en la Facultad de Filosofía y Letras de Universidad de Buenos Aires, 2013. 
accent mark. In the second case, the word carries no accent and it is interchangeable with hasta, incluso or siquiera (until, even, (not) even). Because of its wide variety of functions and semantic values, as pointed out much earlier by Bello (\$1217), some grammarians (Pavón Lucero (1999) have included aun in the group of the so called "particles", a group formed by some prepositions, adverbs and conjunctions. Other authors (Sánchez López 1999, Leonetti 2007 y NGRALE 2009) have placed it within the subgroup of focus words, i.e. words that show the sentence information structure. This paper points out that there is no sharp contrast between these two values aunlaun expressing time or concession - and that they actually share a sense of quantification related to the word's incremental character. This shared value is clearly seen in sentences with a comparative structure, in which cases this word can be equally replaced by todavia or incluso.

Key words: particles, focus words, information structure, quantification.

\section{Introducción}

Tanto los diccionarios como las gramáticas tradicionales suelen distinguir entre aún con tilde, con valor temporal y conmutable por todavía (1-2), y otros usos, en los que aun no lleva tilde y puede ser reemplazado por hasta, incluso o siquiera (3-4).

(1) Son las once de la mañana y aún está durmiendo.

(2) No ha puesto su nombre en la lista aún.

(3) Habló tan claro que lo entendieron aun los que no sabían nada sobre el tema.

(4) Aun con las manos atadas a la espalda, es capaz de abrir una caja fuerte.

A pesar de estas diferencias, nuestro rastreo en un corpus de ejemplos recogidos en distintas fuentes ${ }^{2}$ nos ha permitido comprobar la hipótesis preliminar de que las dos variantes de aún no representan un contraste dicotómico, sino que las reúne un valor compartido, de naturaleza cuantificacional, que se vincula con el carácter escalar de esta partícula, el cual ya había sido reconocido por Bello en su gramática (1928 [1847], \$1216):

Aun, adverbio de tiempo, equivalente á todavía ó hasta ahora. De aquí pasó á sugerir una gradación de ideas que, ya expresa, ya tácita, termina en la palabra ó frase á que lo anteponemos: "Conmovióse al verle, y aun se le arrasaron los ojos de lágrimas".

2. El corpus para este trabajo está constituido por ejemplos extraídos de la prensa escrita de la Argentina (Página 12, La Nación), de la prensa en línea (Infobae, Perfil), de la web y de algunos diccionarios como el VOX, el Diccionario de la Real Academia Española (DRAE), el Diccionario del español actual (DEA) y el Diccionario de partículas discursivas del español (DPDE). Los ejemplos fueron seleccionados en función de los valores semánticos de aún y de su posibilidad de sustitución por otros focalizadores. 
A fin de observar mejor las similitudes y diferencias entre ambas variantes de aún, en este artículo nos centramos en ciertos casos, como los presentados a continuación, en los que aún incide sobre una comparación y resulta posible tanto la conmutación por todavía como por incluso ${ }^{3}$.

(5) El ajedrecista alemán es aún/incluso/todavía mejor jugador que su compañero ruso.

(6) Si no pones interés, te resultará aún más difícil.

(7) Juliana era aún más hermosa que su madre.

(8) Estos barrios tienen su propia seguridad privada y su propia realidad, por lo tanto se achica aún más el territorio que lo que nosotros estamos cubriendo con la presencia de la policía de la provincia de Buenos Aires. Es dos mil veces más chico un territorio que el otro. (Página 12, 20/08/13.)

Según mostraremos en el desarrollo de este trabajo, el estudio del corpus evidencia que aún puede aplicarse a tres ámbitos diferentes que mencionamos seguidamente:

- el temporal en que se origina (v. ap. 3),

- el aspectual (v. ap. 4)

- el modal-comparativo (v. ap. 5).

Por tanto, aún se ha extendido a otros dominios en los que se han diferenciado ciertas presuposiciones derivadas del valor cuantificacional al que esta partícula hace referencia. No obstante, pese a sus "desplazamientos semánticos", según planteamos, en todos sus usos aún retiene un valor básico de 'marcador de límite', que es el que los unifica.

Este artículo consta de las siguientes partes: en primer lugar, presentamos una revisión del tratamiento de aún en ciertos diccionarios del español; en segundo lugar, planteamos cómo, a partir del carácter escalar que manifiesta esta partícula, se agrega al significado temporal de origen un valor enfático de focalización; en tercer lugar, nos referimos al uso de aún con sentido aspectual, como adverbio de fase, que comparte con el empleo anterior el valor de 'límite'. Finalmente, en el último apartado, tratamos un aspecto de aún que, hasta donde sabemos, no ha sido considerado en la bibliografía sobre el tema: se trata de aquellos casos en que este focalizador está en un contexto comparativo y resulta equivalente a todavía y a incluso, es decir, que integra valores de los distintos ámbitos en los que se emplea. En el final presentamos nuestras conclusiones.

3. Según consigna la Ortografía de la lengua española (2010) el adverbio aún/aun puede pronunciarse en español como una palabra con hiato [a.un] o como una átona con diptongo [aun]. El texto académico agrega que el adverbio aún es una palabra tónica generalmente cuando puede reemplazarse por todavía, con valor temporal o ponderativo. Y es una palabra átona y se escribe sin tilde, cuando tiene valor inclusivo y puede ser reemplazado por incluso, hasta o cuando tiene valor concesivo, en esos casos admite la paráfrasis por aunque o a pesar de. Cabe señalar que en la mayoría de los ejemplos extraídos del corpus aún aparece con tilde. 


\section{Aún en los diccionarios del español}

Aún proviene del antiguo adverbio temporal latino adhuc 'todavía, hasta ahora'. En su diccionario etimológico, Corominas ([1961] 2008, p. 53) señala que en el siglo XIII se encuentran las formas adú y ahú, que por analogía con otras palabras terminadas en - $n$ como bien, non, según adoptó la forma aún. Por su parte, en el Diccionario de construcción y régimen de Cuervo (1998, p. 779-783), aún con tilde y aun sin tilde aparecen en la misma entrada, en la que se recoge el valor temporal etimológico 'hasta aquí, hasta ahora' como primera acepción. En una segunda acepción, siguiendo a Bello (1928 [1847]), Cuervo 1998 destaca el valor enfático de esta partícula y plantea que se antepone a una palabra o frase para "representarlas como término final de una gradación expresa o tácita". Respecto de la ortografía, critica que "se nota gran descuido en las ediciones modernas con respecto á la acentuación de este vocablo: no debe tildarse la $u$ sino cuando, cargando sobre ella la fuerza de la pronunciación se profiere el adverbio en dos sílabas: a-ún" (p. 783).

En cuanto a diccionarios generales actuales, en la entrada correspondiente, el DRAE (2015, p. 241), además del temporal, reconoce valores concesivos y de encarecimiento o ponderación. Según los casos, tal como muestra la siguiente entrada que transcribimos, aún es equivalente a todavía (acepciones 1-3), a hasta e incluso (acepción 4) o a siquiera (acepción 5), y en todas ellas funciona como adverbio.

aun

Del lat. adhuc.

Escr. con acento en aceps. 1-3.

1. adv. todavía (\| hasta un momento determinado). Aún estás a tiempo.

2. adv. todavía (\| no obstante, sin embargo). Era quien más espacio tenía y aún protestó.

3. adv. todavía (\| denota encarecimiento o ponderación). Alcanzó más prestigio aún que su padre. Sus palabras introdujeron aún más confusión.

4. adv. Hasta o incluso. Te daré 100 dólares, y aun 200, si los necesitas. Aun estando casados, dormían en habitaciones separadas.

5. adv. siquiera (\| tan solo). No tengo yo tanto, ni aun la mitad.

El diccionario VOX (2001, p. 182) consigna aún como adverbio y también como preposición, en una entrada más completa respecto de la del DRAE y que, además, aporta abundante ejemplificación. En primer lugar, este diccionario da cuenta de las acepciones equivalentes a incluso, inclusive y hasta, alguna de las cuales pueden tener valor hipotético, como en los siguientes ejemplos:

(9) Aun cansado lo hacía mejor que tú.

(10) Aun a disgusto vendrás conmigo a casa de mis padres. 
En segundo lugar, el VOX reseña empleos en los que aún es sinónimo de todavía, como se observa a continuación:

(11) Lo siento pero el doctor aún no ha llegado.

Sin embargo, en algunos de los casos que presenta este diccionario aún no solo admite conmutación por todavía, sino también podría ser reemplazado por incluso, como en (12):

(12) Si vamos en avión, el viaje aún será más caro.

En cuanto al DEA (2011, p. 521), en primer lugar consigna indicaciones respecto de la pronunciación y de la ortografía indicando que "generalmente se pronuncia monosílabo, /áun/, cuando aparece delante del elemento al que se refiere, y bisílabo /aún/ cuando va detrás. En las acepciones 1, 2, 4 y 6 se escribe con tilde AÚN"4. Además de las acepciones 1-7 en las que se plantea su función adverbial, el DEA también consigna a aun como preposición, equivalente a incluso y como conjunción en su uso junto con cuando. Lo que resulta interesante respecto de nuestro trabajo es que el DEA es el único que recoge el hecho de que aun puede acompañar a una palabra comparativa "(más, menos, mejor) para denotar que en el objeto base de la comparación se encuentra ya en grado notable la cualidad o la intensidad de que se habla" (acepción 2) (ejemplo 13). Asimismo, en relación con el valor escalar de este adverbio, el DEA al plantear la equivalencia con incluso, señala que frecuentemente indica que la palabra o sintagma a la que se aplica "supone un grado alto o superior a lo dicho anteriormente" (acepción 3) (ejemplo 14). La acepción 4 también consigna un valor aditivo-adversativo, que retomaremos más adelante, en el subapartado 5.1, en relación con las escalas aditivas (Portolés 2007). Los ejemplos siguientes han sido tomados del DEA:

(13) Al otro día tardé aún más, y de este modo,... fuimos retrasando la clase.

(14) El proyecto fue bien acogido, aun en su forma.

(15) Tenían vendido el caserón en ochenta y cinco millones. Y aún refunfuñaban y maldecían a la curia.

\footnotetext{
4. La acepción 1 es la temporal, según la cual aun "denota persistencia de un hecho hasta el momento en que se habla o de que se habla"; la acepción 2 incluye los casos en que aun acompaña a una palabra comparativa (que retomaremos más adelante en el apartado 5), la 4 se refiere al valor aditivo-adversativo (que veremos en el apartado 5.1) y la 6 es aquella en la que aun equivale a al menos y siquiera. A estas se agregan la acepción 3, en que aun equivale a incluso; la 5 , en que, seguido de un adverbio o de un gerundio, tiene valor adversativo, y la 7 , en que corresponde a ni.
} 


\section{De adverbio temporal a focalizador}

A partir de lo que recogen los diccionarios se puede plantear que el antiguo adverbio temporal latino adhuc hacía referencia a 'lo que ha durado hasta el momento presente o hasta otro punto determinado en el pasado o futuro'. Luego, como se observa en (16) y (17), este adverbio sufrió un proceso de ampliación de su significado, de carácter metafórico, que lo llevó a expresar la idea de 'límite', pero en una serie o sucesión de eventos o estados.

(16) Había resuelto no ceder, arriesgarlo todo y aun perecer si fuese necesario. (Bello 1928 [1847], \$1216.)

(17) Juan enmudeció, palideció y aun lloró de rabia. (Elvira 2009, p. 184.)

Se trata de un pasaje del ámbito temporal al factual, más abstracto, el cual ya había sido reconocido por Bello que, como dijimos, asimilaba hasta con aun. Para este autor, aun en este sentido de gradación pertenece a un conjunto de elementos gramaticales a los que llama cuasiafijos porque pueden anteponerse a toda clase de palabras "modificando su significado y sirviendo como de partículas prepositivas" (Bello 1928 [1847], \$1217).

Asimismo, esta multiplicidad de funciones y valores semánticos de aún, llevó a algunos autores a incluirlo en el grupo de las denominadas "partículas", que engloba preposiciones, adverbios y conjunciones. Como sostiene Pavón Lucero (1999, \$9.1):

La preposición, el adverbio y la conjunción presentan una serie de características comunes que han llevado a incluirlos tradicionalmente en una misma metaclase: la de las partículas.

Las características compartidas por los miembros de este grupo son: a) se trata de palabras invariables; b) establecen relaciones entre oraciones o partes de oraciones; c) generalmente encabezan o nuclean complementos circunstanciales (Ocurrió a las cuatrol entonces/ mientras estábamos de viaje) ${ }^{5}$.

Por su capacidad de incidir sobre distintos tipos de sintagmas, algunos autores como Sánchez López (1999), Leonetti (2007), o Nueva gramática de la lengua española (NGRALE) (2009) han propuesto distinguir dentro de los adverbios, la subclase de los focalizadores, cuya función es poner de relieve la estructura informativa de distintos constituyentes de la oración. Así, Sánchez López (1999, p. 1105) los incluye en el grupo

5. Limitamos nuestro tratamiento de las partículas a su caracterización como metaclase de palabras en la que aun, como adverbio, puede considerarse incluido, junto con las preposiciones y las conjunciones. Según consideramos, su tratamiento como marcadores del discurso (Zorroaquino y Portolés 1999) excede el marco más acotado de este trabajo. 
de cuantificadores focales o presuposicionales, puesto que "inducen la interpretación cuantitativa del elemento al que modifican por implicación de existencia (o inexistencia) de otros elementos. En su interpretación lógica interviene el cuantificador existencial, lo que los relaciona con los indefinidos no universales". Para esta autora, los cuantificadores presuposicionales agregan a su sentido exclusivo o inclusivo un matiz valorativo o evaluativo.

Por su parte, para Leonetti (2007), los distintos focalizadores pueden adjuntarse a cualquier tipo de sintagma para incidir sobre él, ya se trate de un sintagma preposicional, adjetival o nonimal o incluso de un sintagma verbal o de complementante. A su vez, Bosque y Gutiérrez Rexach (2009) denominan a palabras como sólo, incluso, hasta, además, etc., que se corresponden con operadores en la FL (OPERADORES DE FOCO), ya que tienen la peculiaridad de asociarse con el constituyente focalizado.

Si bien la NGRALE (2009) considera dentro de los focalizadores de inclusión ${ }^{6}$ solamente incluso, hasta y ni siquiera, como hemos sugerido en trabajos anteriores (Ferrari, Giammatteo y Albano 2011 y Giammatteo, Ferrari y Albano 2012), aun podría ubicarse en el mismo subgrupo, dado que puede ocupar, en ciertos casos, contextos equivalentes a los de estos focalizadores, como se observa a continuación:

(18) Aun/hasta/incluso/siquiera el más pequeño ruido lo perturbaba.

(19) El sueldo no nos alcanza ni siquieralaun para pagar la luz.

(20) Hace frío aun/hasta/incluso con la calefacción encendida.

Según ya hemos planteado (Ferrari, Giammatteo y Albano 2011), el carácter escalar o gradual es el que unifica al conjunto de los focalizadores. En un trabajo posterior, hemos avanzado un poco más sobre nuestros planteos anteriores para postular que los focalizadores estudiados constituyen una clase de palabra independiente, de tipo funcional ${ }^{7}$. Particularmente, adaptando una propuesta de Fauconnier (1975) para el análisis de los superlativos, también retomada por Portolés (2007), hemos propuesto que la noción central que guía el comportamiento de los focalizadores es un principio escalar regido por una escala pragmática ${ }^{8}$. Estas escalas se basan en un tópico compartido y los sintagmas que las constituyen se pueden ordenar según su fuerza informativa (Portolés,

6. La NGRALE (2009) reconoce cuatro grupos principales de focalizadores: 1) exclusivos -solamente, únicamente-; 2) incluyentes -incluso, hasta, ni siquiera-; 3) identificativos-exactamente, justamente-; 4) particularizadores-especialmente, sobre todo-.

7. Es decir, estas palabras han pasado de pertenecer a distintas clases de palabras léxicas, a constituir una clase de palabras funcional, de significado fundamentalmente gramatical o relacional y con implicaciones pragmáticas, ya que la interpretación de muchos de los valores que presentan depende del contexto en que se insertan. Para los detalles de este planteo remitimos a Giammatteo, Ferrari y Albano (2012).

8. Portolés (2007, p. 137) define las escalas pragmáticas como aquellas que "nacen de nuestro conocimiento del mundo". 
2007). Por tanto, implican que si un enunciado es verdadero respecto del elemento ubicado en la posición inferior, también es verdadero respecto de todos los elementos contenidos en ella. Así, según nuestros planteos, si aplicamos estos conceptos, en una oración como (21) es posible distinguir:

- El contenido proposicional aseverado: los críticos más severos han elogiado su obra.

- El significado aportado por el focalizador: no solo los críticos más severos han elogiado su obra (contenido proposicional), sino también otros lo hicieron (implicación).

- Las inferencias pragmáticas derivadas del valor escalar del focalizador:

- la contraexpectativa: no se esperaba que los críticos más severos elogiaran su obra; y

- la inclusividad en el grado mínimo de la escala: los críticos más severos eran los últimos que se esperaba que elogiaran su obra.

(21) Aun/incluso los críticos más severos han elogiado su obra.

En suma, la función de los focalizadores de inclusión estudiados consiste en señalar la existencia de una escala pragmática de probabilidad con respecto al contexto en que aparece el enunciado. Uno de los extremos de la escala es precisamente el sintagma al que modifica aun -incluso [los críticos más severos]-. Se afirma que los críticos más severos elogiaron algo y se presupone toda la escala. La presuposición se convierte en una inferencia pragmática de la aserción Los críticos elogiaron su obra, que nos coloca en una hipotética escala de probabilidad (era muy poco probable que los críticos más severos elogiaran su obra) y, justamente, lo que el focalizador destaca es esta presuposición.

En relación con los tres contenidos semánticos que suelen asociarse con el foco (Bosque y Gutiérrez Rexach 2009), el focalizador:

- Resalta el constituyente focalizado: También los críticos más severos elogiaron su obra $\rightarrow$ Énfasis.

- Lo incluye entre los elementos de la escala pragmática implicada: Hasta los críticos más severos elogiaron su obra / Todos y también los críticos más severos la elogiaron $\rightarrow$ Exhaustividad.

- Lo contrasta con los otros componentes de dicha escala: No solo los demás, sino también los críticos más severos elogiaron su obra $\rightarrow$ Alternatividad.

\section{Aún como adverbio aspectual}

Además del significado temporal, García Fernández (1999) reconoce un valor aspectual para aún, al que ubica en el grupo de los adverbios que manifiestan "fase", integrado por ya, todavía y sus correspondientes formas negativas ya no y todavía no, que "indican diferentes fases en el desarrollo de un evento" (\$48.1.2). El autor aclara que se trata de fases "sucesivas" (\$48.1.2.3.) y ejemplifica con El arroz ya está cocido, en que 
interpretamos que en una fase anterior "el arroz no estaba cocido". Siguiendo a Muller, (1975, cit. en García Fernández), el autor plantea que todavía, ya y sus respectivas negaciones "presuponen una fase anterior al período focalizado o enfocado por el aspecto y han de permitir una posible (pero no obligatoria) fase sucesiva” (\$48.1.2.3.)”

A partir del planteo de García Fernández (1999), hemos elaborado la siguiente tabla, que despliega los valores de fase vinculados con ya, todavía y sus respectivas negaciones.

TABla I: VAlORES DE FASE

\begin{tabular}{|l|l|l|l|l|}
\hline & Fase previa & Fase afirmada & $\begin{array}{l}\text { Posible fase } \\
\text { posterior }\end{array}$ & Ejemplo \\
\hline Todavía no & $\mathrm{Neg}$ & $\mathrm{Neg}$ & Af. & 1. El nińo todavía no está curado. \\
\hline Ya & $\mathrm{Neg}$ & Af & Af & 2. Juan ya tiene coche. \\
\hline Todavía & Af & Af & Neg & $\begin{array}{l}\text { 3. Los invitados todavía } \\
\text { están en la iglesia. }\end{array}$ \\
\hline Ya no & Af & Neg & Neg & 4. María ya no trabaja aquí. \\
\hline
\end{tabular}

De acuerdo con lo propuesto en la Tabla 1, con todavía no decimos que un evento, que no ha ocurrido (fase previa), continúa sin ocurrir (fase afirmada), pero podría hacerlo (fase posterior). En cambio, con la correspondiente forma afirmativa -todavíalo que decimos es que un evento en desarrollo (fase previa) continúa (fase afirmada), aunque posiblemente no siga (fase posterior). Cuando usamos ya se señala un cambio y se indica que un evento que no se daba (fase previa), ha comenzado (fase afirmada) y posiblemente continúe (fase posterior); y cuando se utiliza la forma negativa -ya no- se señala que un evento en desarrollo (fase previa) ha cesado (fase afirmada) y que es posible que esa situación se mantenga (fase posterior). En este sentido se puede decir que dado que la fase previa y la afirmada coinciden, todavía tiene valor durativo; mientras que con $y a$, en que estas dos fases tienen siempre signo opuesto, el señalamiento es puntual y se enfatiza el momento en que se produce el cambio.

Por su parte, De Miguel y Fernández Lagunilla (1999) consideran a solo y a aún como operadores que permiten mostrar la estructura interna de los eventos. Para aún presentan oraciones que contrastan, como las siguientes:

(22) María aún estudia.

(23) *Juan aún sale de la fiesta. 
La incompatibilidad de (23) se explica diciendo que aún constituye un operador aspectual que enfoca la fase intermedia de un evento, "de manera que presupone que existe una fase anterior en la que el evento ya se daba -y probablemente habrá una fase posterior, aunque esto no está implicado directamente" (p. 19). Por tanto, para las autoras, aún es compatible con estudiar, que es una actividad, pero no con salir, que denota un evento ingresivo de logro; y por tanto, carente de fase anterior'.

En relación con nuestros planteos, lo que resulta pertinente de ambos enfoques es que al vincular estas partículas con el valor aspectual de fase destacan que los focalizadores hacen referencia a un límite. Como muestran los ejemplos (24) y (25), en el caso de aun se trata de un límite en el interior del evento, del cual es posible derivar su valor escalar y gradual, como veremos en el próximo apartado.

(24) Aún tengo al sol para besar tu sombra (Letra de la canción Ángel eléctrico -Soda Stereo- rock.com.ar).

(25) Lionel Messi aún no olvida la final de la Copa Améica ante Chile (www.emol.com/ noticias/Deportes/2015/12/13).

\section{Aún en la escala comparativa}

Si ahora pasamos a considerar el comportamiento de aún en el último ámbito propuesto: el modal-comparativo, la pregunta que podemos hacernos es por qué esta partícula puede ser simultáneamente reemplazada por todavía, que tiene valor temporoaspectual y por incluso, que es un focalizador.

Desde el punto de vista de su conformación, las estructuras comparativas exigen la presencia a la derecha del núcleo del sintagma de un complemento denominado coda que, semánticamente, "no hace referencia ni a individuos ni a propiedades sino a 'grados" (Sáez del Álamo 1999, §17.1). Así, en una oración como (26) la coda que Luis significa el grado hasta el que Luis es alto o el grado de altura de Luis, lo que quiere decir que aquí también estamos en un ámbito escalar. Para Sáez del Álamo, el sintagma comparativo tiene carácter existencial, por lo que (26) se interpreta como 'existe un grado, superior al grado de altura de Luis, tal que Juan es alto hasta ese grado' (según se muestra en la Figura 1).

(26) Juan es más alto que Luis.

(= que el grado de altura de Luis)

9. En cambio, aún sí es posible si el evento se interpreta con valor iterativo-Aún salía gente de la fiesta-, o si se trata de un nombre no contable-Aún sale agua de la canilla. 


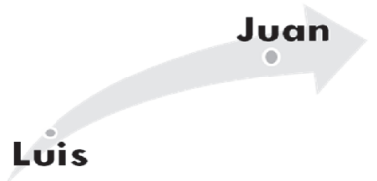

Figura 1. Estructura comparativa

Según Sánchez López (2006), el grado denotado por las estructuras comparativas se define por su relación con el grado de referencia manifestado por la coda comparativa. La relación entre ambos grados puede ser de superioridad (más), de inferioridad (menos) o de igualdad (tan). La relación entre los dos puntos en la escala, en los dos primeros casos (superioridad e inferioridad), delimita un intervalo que se puede medir o valorar y que se denomina diferencial, porque cuantifica la diferencia que hay entre los dos grados comparativos. Como se muestra en (27), la expresión de esa medida se puede señalar mediante una expresión de cantidad que antecede al elemento en grado comparativo.

(27) Juan es tres centímetros/ un poco más alto que Luis.

Ahora bien, en relación con los planteos considerados, nuestra propuesta es que, además de cuantificarse, ese intervalo puede ser ponderado (ya sea en una comparación de superioridad o de inferioridad) si se le añade un focalizador como aún, como se ejemplifica en (28).

(28) Juan es aún más alto que Luis.

En estas construcciones comparativas, el focalizador aporta simultáneamente valores provenientes de sus distintos ámbitos de inserción, lo que, según creemos, permite explicar por qué en estos casos aún puede ser reemplazado tanto por todavía como por incluso. Por consiguiente, los valores que aún manifiesta son los siguientes:

- Valor de fase. En relación con la escala establecida, que en este caso es de superioridad, el focalizador indica que la altura de Juan corresponde a una fase afirmada que supera (aunque también continúa) la fase o nivel inferior en la que se ubica la altura de Luis, que correspondería a la fase previa, según lo señalado en el ámbito eventivo (v. ap. 4). A este valor de continuidad es al que apunta la posible paráfrasis con todavía (29). 
(29) Juan es todavía más alto que Luis.

- Valor focalizador. En relación con este ámbito, aun aporta los valores siguientes:

- Énfasis sobre el constituyente al que se aplica, en este caso el sintagma comparativo

$\rightarrow$ Aunque Luis posee altura (es alto), Juan lo supera (en cuanto al grado en que posee esta propiedad).

- Exhaustividad, que implica la inclusión dentro de la escala gradual involucrada

$\rightarrow$ Tanto Luis como Juan poseen altura, pero además Juan posee la propiedad hasta un nivel superior.

- Alternatividad, que seńala el contraste entre ambos grados de la propiedad: el grado $x$, correspondiente a 'la altura de Juan', y el grado $y$, correspondiente a la altura de Luis, comparativamente menor

$\rightarrow$ No solo Luis es alto, sino que también Juan lo es y en mayor medida. Esta exigencia de contraste explica que el focalizador no pueda aplicarse a las comparativas de igualdad (30).

(30) *Juan es aún tan alto como Luis.

Así, según postulamos en este trabajo, en función de este valor focalizador, aún también puede ser reemplazado por incluso en las construcciones comparativas (31).

(31) Juan es incluso más alto que Luis.

\subsection{La presuposición pragmática. El uso argumentativo}

En estos casos de focalización sobre el sintagma comparativo también existe una presuposición de tipo pragmático. Así, en un ejemplo como (26) (repetido aquí como (26bis)), en que no hay focalizador, la coda comparativa -que Luis-, denota un grado de referencia relativo y no implica ni la afirmación ni la negación de la propiedad, ni tampoco la adecuación a una norma estándar externa.

(26bis) Juan es más alto que Luis.

Es decir, a pesar de que la cualidad involucrada es la altura, ni Juan ni Luis deben ser necesariamente altos, pueden incluso ser dos enanos (respecto de la norma) y la altura del primero -Juan - superar a la del segundo -Luis-.

En cambio, cuando se añade un focalizador se presupone la afirmación o la negación de la propiedad de referencia: por lo que en (28), repetido como (28bis), entendemos que Luis es efectivamente alto de acuerdo a una norma establecida y que Juan lo supera en altura. 
(28bis) Juan es aún más alto que Luis.

De modo semejante sucede en (32), en que se comparan dos estados sucesivos de delgadez del mismo objeto y en (33), en que se comparan dos entidades diferentes en cuanto al grado de importancia que se les asigna.

(32) Apple anuncia su nuevo Macbook de 12" aún más delgado que el Macbook Air. (http://cincodias.com/cincodias/2015/03/09/gadgets/1425922605_621018.html. Acceso: 09/03/15.)

(33) Creo que lo que comemos es importante para todos nosotros y el tipo de alimentación con el que crecen nuestros hijos y nuestros nietos es aún más importante. (Linguee, http://es.bab.la/diccionario/espanol-ingles/es. Acceso: Acceso: 10/ 03/15.)

Por supuesto, como es frecuente en las comparativas, lo dicho se aplica incluso cuando la coda queda implícita, como en los siguientes ejemplos:

(34) Nuestras previsiones para el año próximo muestran que la reactivación en Europa debería ser aún más sólida a condición de que evitemos nuevas crisis políticas y turbulencias en los mercados. (La Nación, http://www.lanacion.com.ar/1610741la-ue-salio-de-la-recesion-mas-larga-de-su-historia/. Acceso: 15/08/13.)

(35) El periodista Josep Martí Gómez considera que «si la Ley de Extranjería de 1985 hecha por los socialistas fue mala, ésta es aún peor y fracasará. (Canarias 7, CREA. Acceso: 5/02/01)

Resulta interesante también destacar que el focalizador aún puede posponerse al primer término de la comparación:

(36) Hay algo que Cristina Kirschner no puede entender: que un intendente la haya derrotado. Si alguien fuera sincero, podría contarle una conclusión de esta semana que significaría una herida más profunda aún en su narcisismo, (La Nación, http:// www.lanacion.com.ar/1611792-viene-de-tapa/. Acceso: 18/08/13.)

(37) Cavallo: "Néstor fue más entusiasta aún que Scioli y que Macri de las privatizaciones". (Perfl.com, https:/www.facebook.com/perfilcom/posts/1021367124550087/.

Acceso: 28/07/15.)

Asimismo, el focalizador puede incidir sobre una forma verbal que lexicaliza un cuantificador de grado comparativo (38) o sobre predicaciones entre las cuales se establece una gradación (39) y (40). 
(38) El relato de la conspiración empeoró aún más la relación maltrecha que ya existía entre Massa y el gobernador Daniel Scioli. (La Nación, http://www.lanacion.com. ar/1798679-daniel-scioli-ignora-a-sergio-massa-y-desafia-a-mauricio-macri-a-undebate-por-tv/. Acceso: 18/08/13.)

(39) Tiene 39 ańos y hace tres ni se le ocurría que algún día se casaría. Y menos aún que en tan poco tiempo iba a recuperarse del alcoholismo, recuperar a su hijo mayor que vivía con el padre, y organizar una familia con seis hijos... (La Nación, http://www. lanacion.com.ar/1612198-terapia-de-rescate-experiencia-inedita-en-una-villa/. Acceso: 20/08/13.)

(40) El exceso de emisión dispararía aún más el precio del dólar. (Infobae, http://www. infobae.com/2015/06/23/1737135-el-exceso-emision-dispararia-aun-mas-elprecio-del-dolar/. Acceso: 23/06/15.)

Desde una perspectiva discursiva, este focalizador ligado al cuantificador puede funcionar como partícula que refuerza la argumentación ${ }^{10}$. Se trata de casos de reformulación en los que el hablante considera insuficiente la fuerza del argumento empleado, por lo que añade otro argumento que facilite al oyente realizar las inferencias necesarias para llegar a la conclusión (Portolés 1998), tal como se ejemplifica en (41).

(41) Soy un completo desastre, más aún soy la máxima expresión de la imperfección vuelta mujer;... (Blog, http://iammafecastro.tumblr.com/post/92300536770/ soy-un-completo-desastre-m\%C3\%A1s-a\%C3\%BAn-soy-la-m\%C3\%A1xima. Acceso: 02/10/16.)

(41) es un claro ejemplo de lo que Portolés denomina una escala aditiva culminativa ${ }^{11}$, ya que no solo se ańade otro argumento, sino que este se presenta como superior, o sea más informativo, que el anterior.

Al respecto, el DPDE (2008) también plantea que más aún, menos aún, aún más o aún menos constituyen partículas discursivas que enfatizan el argumento que introducen. Así, por ejemplo, define más aún como aquella partícula que "presenta el miembro del

10. En este mismo sentido, Portolés (2007, p. 137-138) defiende que dentro de las escalas informativas, además de las semánticas y de las pragmáticas, existe un tercer tipo "que consiste en aquellas convocadas por el significado de procesamiento de una partícula discursiva", las cuales pueden llevar incluso a invertir el ordenamiento proveniente de nuestro conocimiento de mundo:

... le comentamos a un campesino cosechero que, según un antiguo libro, aquel vino de Amandi era el preferido de los emperadores de Roma. Y el hombre respondió con naturalidad: "No me extraña. ¡Si hasta vienen de Vigo a por él!”. (Manuel Rivas. El País Semanal (España), 19/11/1999, p. 128.)

11. Las escalas aditivas son aquellas en las que el valor escalar consiste en la suma de un elemento al valor inferior $(n+1)$. Estas escalas pueden ser culminativas o no (el nuevo elemento solo se ańade a los anteriores, sin constituir un valor superior) (Portolés 2007). 
discurso en que aparece como un argumento añadido más fuerte y que se ha de tener más en cuenta que otro para una conclusión”.

El DPDE presenta el ejemplo siguiente:

(42) El soltero de oro, como se le llamaba últimamente, ha sucumbido a los encantos del amor. Más aún, podría estar pensando en casarse próximamente. (Hola, CREA, 1995.)

En (42), los dos argumentos sirven para llegar a la misma conclusión (el soltero de oro está cambiando sus hábitos), pero el segundo argumento tiene más fuerza que el primero.

En ciertos casos, más aún no agrega un nuevo argumento sino que directamente reemplaza el anterior, lo que en términos de Portolés (2007) constituye una escala de tipo sustitutivo ${ }^{12}$, como en el ejemplo siguiente, también tomado del DPDE:

(43) De hecho, portavoces de diversos centros de fecundación estadounidenses, que en la pasada primavera descartaban absolutamente la clonación en humanos, declaran actualmente que están dispuestos a considerarla. Más aún, no se oculta que en algunos de estos centros están ya en marcha experimentos en este sentido. (Época, CREA, 1993.)

En el caso de menos aún, esta partícula también presenta el argumento que encabeza como el más fuerte o el más adecuado para ser tenido en cuenta, pero a diferencia de más aún debe ir precedido en el primer constituyente por una negación explícita, como se observa en (39) y (44):

(44) La pericia demuestra que nunca existió ese embarazo y menos aún el parto de los mellizos (La Capital, http://www.lacapital.com.ar/la-pericia-demuestra-que-nunca-existio-eseembarazo-y-menos-aun-el-parto-los-mellizos-n448260.html/. Acceso: 28/10/14.)

\section{Conclusiones}

Según hemos mostrado en el desarrollo de este trabajo, la línea entre aún temporal y aun focalizador-comparativo no está todavía nítidamente delineada. El análisis del corpus nos ha permitido concluir que aún posee tres valores diferentes: el temporal, el aspectual y el modal-comparativo. Sin embargo, hemos demostrado que esta partícula posee una

12. “...porque el valor superior reemplaza al inferior, y viceversa” (p. 139). 
significado general de 'marcador de límite', derivado de su valor cuantificacional, que unifica las distintas variantes de aún/aun.

Si bien en algunos contextos los diferentes usos de aún pueden deslindarse claramente, en las comparativas los valores se asocian y conjugan a partir del señalamiento de una fase afirmada/negada (valor de fase) y al mismo tiempo puesta en foco a través del contraste con otra a la que supera en más o en menos y que, en una perspectiva pragmática, presupone lo afirmado (valor focalizador). También, según hemos podido observar, en ciertos casos el focalizador aún se une a un cuantificador, como más o menos, adoptando una función argumentativa en el discurso.

En síntesis, los ejemplos presentados nos han permitido mostrar estas zonas de intersección; no obstante, cuestiones como la posición que el focalizador puede adoptar respecto del término comparativo al que se aplica o la posibilidad de incidir sobre distintos tipos de sintagmas, apenas han podido ser mencionadas; mientras que otras, como la normativa ortográfica respecto de la tilde en estos casos de solapamiento de valores, no han sido tratadas y, según esperamos, podrán ser objeto de futuras investigaciones.

\section{Referencias}

Bello, A. (1928 [1847]). Gramática de la lengua castellana destinada al uso de los americanos. París: A. Blot editor.

Briz, A., Pons, S., y Portolés, J. (eds.) (2008). Diccionario de partículas discursivas del español. Disponible en www.dpde.es. [Consulta 27 de julio de 2015].

Bosque, I., y Gutiérrez Rexach, J. (2009). Fundamentos de sintaxis formal. Madrid: Ediciones Akal, S.A.

Corominas, J. (2008). Breve diccionario etimológico. Buenos Aires: Del Nuevo Extremo; Madrid: Gredos.

Cuervo, R. (1998). Diccionario de construcción y régimen de la lengua castellana. Barcelona: Herder Editorial.

De Miguel, E., y Fernández Lagunilla, M. (1999). El operador aspectual se. Revista Española de Lingüistica, 30(1), 13-43.

DEA = Seco, M., Olimpia, A., y Ramos, G. (2011).

DPDE = Briz, A., Pons, S., y Portolés, J. (2008).

DRAE $=$ Real Academia Española (2014).

Elvira, J. (2009). Evolución lingüistica y cambio sintáctico. Frankfurt am Main: Peter Lang

Fauconnier, G. (1975). Pragmatic scales and logical structure. Linguistic Inquiry, 6(3), 353-37.

Ferrari, L., Giammatteo, M., y Albano, H. (2011). Adverbios operadores de foco: El caso de incluso, hasta, solo y aun. En J. Camacho, M. Luján y L. Sánchez (eds.), 
Estudios de gramática formal del español contemporáneo (p. 30-41). Disponible en http://www.linguisticalfal.org/cuadernos.html. Acceso: 15/09/15.

Ferrari, L., y Giammatteo, M. (en prensa). Aún como marcador de posición extrema. En B. Lavandera (ed.), Actas de las II Jornadas Internacionales. Sociolingüistica y Análisis del Discurso. Buenos Aires: Universidad de Buenos Aires.

García Fernández, L. (1999). Los complementos adverbiales temporales. La subordinación temporal. En I. Bosque y V. Demonte (Eds.), Gramática descriptiva de la lengua española (p. 3129-3207). Madrid: Espasa Calpe.

Giammatteo, M., Ferrari, L., y Albano, H. (2012). Operadores de foco: aspectos léxicosintácticos y procesos de gramaticalización. En M. Giammatteo, L. Ferrari y H. Albano (eds.), Léxico y sintaxis (p. 107-123). Mendoza: Universidad de Cuyo y SAL, Serie Volúmenes Temáticos.

Leonetti, M. (2007). Los cuantificadores. Madrid: Arco/Libros.

Muller, C. (1975). Remarques syntactico-sémantiques sur certain adverbes de temps. Le Français Moderne, 43(1), 12-38.

NGRALE = Real Academia Española y Asociación de Academias (2009).

Pavón Lucero, Ma . V. (1999). Clases de partículas: preposición, conjunción y adverbio. En I. Bosque y V. Demonte (eds.), Gramática descriptiva de la lengua española (p. 565-655). Madrid: Espasa Calpe.

Portolés, J. (1998). La Teoría de la Argumentación en la Lengua y los marcadores del discurso. En M. A. Zorraquino y E. Montolío (eds.) Los marcadores del discurso. Teoría y análisis (p. 71-91). Madrid: Arco/Libros.

Portolés, J. (2007). Escalas informativas aditivas. Spanish in Context, 4(2), 135-157.

Real Academia Española (2010). Ortografía de la lengua española. Madrid: Espasa Calpe. Real Academia Española (2014). Diccionario de la lengua española (23ª edición). Madrid: Espasa.

Real Academia Española y Asociación de Academias (2009). Nueva gramática de la lengua española (Sintaxis II). Madrid: Espasa Libros.

Sáez del Álamo, L. (1999). Los cuantificadores: Las construcciones comparativas y superlativas. En I. Bosque y V. Demonte (eds.), Gramática descriptiva de la lengua española (p. 1129-1179). Madrid: Espasa Calpe.

Sánchez López, C. (1999). Los cuantificadores: clases de cuantificadores y estructuras cuantificativas. En I. Bosque y V. Demonte (eds.), Gramática descriptiva de la lengua española (p. 1025-1128). Madrid: Espasa Calpe.

Sánchez López, C. (2006) El grado de adjetivos y adverbios. Madrid: Arco/Libros.

Seco, M., Olimpia, A., y Ramos, G. (2011). Diccionario del español actual. Madrid: Aguilar.

VOX (2001). Diccionario de la lengua española. Lema. Barcelona: Spes. 
Zorroaquino, J., y Portolés, J. (1999). Los marcadores del discurso. En I. Bosque y V. Demonte (eds.), Gramática descriptiva de la lengua española (p. 4051-4213). Madrid: Espasa Calpe. 